Check for updates

Cite this: Lab Chip, 2018, 18, 3225

Received 27th April 2018,

Accepted 16th July 2018

DOI: $10.1039 / c 8 l c 00438 b$

rsc.li/loc

\section{Biomolecular recognition on nanowire surfaces modified by the self-assembled monolayer $\dagger$}

\author{
Taisuke Shimada, iD *ab Takao Yasui, iD *abc Asami Yokoyama, ${ }^{a}$ Tatsuro Goda, iD d \\ Mitsuo Hara, (iD e Takeshi Yanagida, ${ }^{\text {gg }}$ Noritada Kaji, ${ }^{\text {h }}$ Masaki Kanai, ${ }^{f}$ \\ Kazuki Nagashima, ${ }^{f}$ Yuji Miyahara, ${ }^{d}$ Tomoji Kawai ${ }^{9}$ and Yoshinobu Baba*abi
}

\begin{abstract}
Molecular recognition is one of the key factors in designing biosensors due to which nanowires functionalized with molecular recognition have attracted a lot of attention as promising candidates for nanostructures embedded in biosensors. However, the difficulty in real-world applications with analytical specificity is that molecular recognition on nanowires mainly depends on antibody modification with multistep modification procedures. Furthermore, the antibody modification suffers from nonspecific adsorption of undesired proteins in body fluid on the nanowires, which causes false responses and lowers sensitivity. Herein, we propose biomolecular recognition using surface-modified nanowires via thiolated 2-methacryloxyethyl phosphorylcholine (MPC$\mathrm{SH}$ ). MPC-SH enables self-assembled monolayer (SAM) modification, which contributes to the reduction of nonspecific adsorption of biomolecules onto the nanowires, and the specific capture of a target protein is attained in the presence of calcium ions. Our concept demonstrates the recognition of the biomarker protein on nanowire surfaces modified by MPC-SH SAM with a single step modification procedure.
\end{abstract}

\footnotetext{
${ }^{a}$ Department of Biomolecular Engineering, Graduate School of Engineering, Nagoya University, Furo-cho, Chikusa-ku, Nagoya 464-8603, Japan.

E-mail: shimada.taisuke@c.mbox.nagoya-u.ac.jp, yasui@chembio.nagoya-u.ac.jp, babaymtt@chembio.nagoya-u.ac.jp

${ }^{b}$ ImPACT Research Center for Advanced Nanobiodevices, Nagoya University, Furocho, Chikusa-ku, Nagoya 464-8603, Japan

'Japan Science and Technology Agency (JST), PRESTO, 4-1-8 Honcho, Kawaguchi, Saitama 332-0012, Japan

${ }^{d}$ Institute of Biomaterials \& Bioengineering, Tokyo Medical and Dental University (TMDU), 2-3-10 Kanda-Surugadai, Chiyoda, Tokyo 101-0062, Japan

${ }^{e}$ Department of Molecular and Macromolecular Chemistry, Graduate School of Engineering, Nagoya University Furo-cho, Chikusa-ku, Nagoya 464-8603, Japan

${ }^{f}$ Institute for Materials Chemistry and Engineering, Kyushu University, 6-1 Kasuga-koen, Kasuga, Fukuoka 816-8580, Japan

${ }^{g}$ The Institute of Scientific and Industrial Research, Osaka University, 8-1 Mihogaoka, Ibaraki, Osaka 567-0047, Japan

${ }^{h}$ Department of Chemistry and Biochemistry, Graduate School of Engineering, Kyushu University, Moto-oka 744, Nishi-ku, Fukuoka 819-0395, Japan

${ }^{i}$ Health Research Institute, National Institute of Advanced Industrial Science and Technology (AIST), Hayashi-cho, Takamatsu, Kagawa 761-0395, Japan

$\dagger$ Electronic supplementary information (ESI) available. See DOI: 10.1039/ c8lc00438b
}

\section{Introduction}

Molecular recognition is one of the key factors in biosensor design, and it is realized via specific biomolecular interactions. ${ }^{1}$ Biosensors are used to diagnose health conditions via sensor biomolecules, ${ }^{2,3}$ and their concentrations in biological fluids are indicators of cancer, inflammation and lifestyle diseases. Since biosensors detect targeted biomarker molecules by converting binding events into signals, molecular recognition function on a sensing part is essential for analytical specificity. In recent years, nanostructure-embedded biosensors have been developed towards miniaturization, label-free detection and high sensitivity. ${ }^{4-7}$ Accordingly, nanostructures with molecular recognition functions have become more important. ${ }^{8-10}$ However, the difficulty in real-world applications with analytical specificity is that molecular recognition on the nanostructures mainly depends on antibody modification via multistep modification procedures. Furthermore, the antibody modification suffers from nonspecific adsorption of

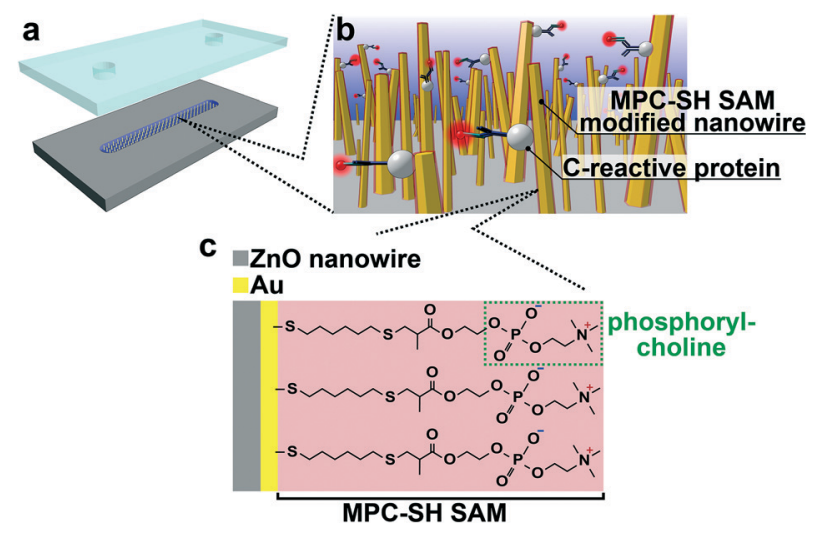

Fig. 1 (a) An illustration of MPC-SH SAM-modified nanowires in a microchannel. (b) An illustration of CRP recognition of MPC-SH SAMmodified nanowires. (c) Molecular formula of MPC-SH SAM on the Ausputtered nanowires. Phosphorylcholine groups specifically interact with CRP in the presence of calcium ions. 
undesired proteins in body fluids on the nanostructures, ${ }^{11-13}$ which leads to false responses and decrease in sensitivity. ${ }^{14} \mathrm{~A}$ molecular recognition methodology for the capture of a target analyte on nanostructure surfaces instead of antibodies is desired.

Herein, we propose a biomolecular recognition methodology using thiolated 2-methacryloxyethyl phosphorylcholine (MPC-SH)-modified nanowires for the capture of a target analyte on the nanowire surface instead of primary antibodies (Fig. 1). Nanowires were chosen because they are promising candidates as nanostructure-embedded biosensors with the advantage of a high surface-to-volume ratio. ${ }^{15-22}$ The nanowires were treated by MPC-SH, which enabled the spontaneous formation of a self-assembled monolayer (SAM) in a single step process. Generally, SAM modification provides covalent coatings on noble metal surfaces, which have higher thermal and chemical stabilities in comparison with antibody coatings. Furthermore, MPC-SH SAM can reduce nonspecific adsorption of biomolecules. In the presence of calcium ions, the specific capture of human C-reactive protein (CRP) can be achieved via the zwitterionic phospholylcholine (PC) group present in MPC-SH. ${ }^{23-29} \mathrm{CRP}$ is a general nonspecific inflammatory biomarker protein, and its systemic concentration dramatically increases 100 to 1000 times during the acute phase of inflammation and infection. Therefore, CRP detection can be used directly to assess disease initiation, severity, and progression. ${ }^{30-36}$ In this study, we demonstrate that MPC-SH SAM-modified nanowires show specificity in biomolecular recognition for CRP detection.

\section{Experimental}

\section{Fabrication of MPC-SH SAM-modified nanowires inside a microchannel}

A microchannel $(W, 200 \mu \mathrm{m} ; L, 1.5 \mathrm{~cm} ; D, 22 \mu \mathrm{m})$ was fabricated by conventional photolithography and deep reactive ion etching (DRIE) techniques. 1,1,1,3,3,3-Hexamethyldisilazane (OAP, Tokyo Ohka Kogyo Co., Ltd.) and OFPR8600 (Tokyo Ohka Kogyo Co., Ltd.) were spin-coated on an Si substrate (Advantech Co., Ltd.), and the microchannel pattern was formed by photolithography. The microchannel was etched using a DRIE apparatus (MUC-21, Sumitomo Precision Production Co., Ltd.). The DRIE process consisted of the following steps, which were repeated 30 times: isotropic etching by $\mathrm{SF}_{6}$ plasma; forming a fluorocarbon sacrificial layer by $\mathrm{C}_{4} \mathrm{~F}_{8}$ plasma; and anisotropic etching by $\mathrm{Ar}$ plasma. Then, MPC-SH SAM-modified nanowires were fabricated inside the microchannel. For the fabrication of $\mathrm{ZnO}$ nanowires, a 100 $\mathrm{nm} \mathrm{ZnO}$ layer was formed using a sputtering apparatus (SC701Mk Advance, Sanyu Electron Co., Ltd.) on the bottom of the microchannel surface, and it was used as a template for ZnO nanowires during hydrothermal synthesis. Next, the substrate with the microchannel having the $\mathrm{ZnO}$ layer was immersed into the nanowire hydrothermal growth solution (40 $\mathrm{mL}$ ), which was a mixture of $40 \mathrm{mM}$ hexamethylenetetramine (HMTA, Wako Pure Chemical Industries, Ltd.) and $40 \mathrm{mM}$ zinc nitrate hexahydrate (Thermo Fisher Scientific Inc.); this mixture was heated at $95{ }^{\circ} \mathrm{C}$ for 3 hours. ${ }^{37,38}$ After washing with pure water, an Au layer was sputtered on the $\mathrm{ZnO}$ nanowires to obtain SAM. The Au sputtering at a rate of $89 \mathrm{~nm}$ $\min ^{-1}$ on a flat substrate was performed for $60 \mathrm{~s}$. The positive photoresist layer was removed by immersing the substrate with the Au-coated nanowires and microchannel into Stripper104 (Tokyo Ohka Kogyo Co., Ltd.) at $75{ }^{\circ} \mathrm{C}$ for $30 \mathrm{~min}$. Next, MPC-SH was dissolved in pure water, and the substrate with the Au-coated nanowires and microchannel was put into this MPC-SH solution for $1 \mathrm{~h}$ at room temperature and in the dark. After that, the substrate was removed from the solution and washed using pure water. The substrate with the Aucoated nanowires and microchannel was covered with a polydimethylsiloxane (PDMS) sheet. The PDMS sheet was prepared using Silpot184 (Dow Corning Toray Co., Ltd.) and Catalyst Silpot184 (Dow Corning Toray Co., Ltd.), and it had a $\varphi 1.5 \mathrm{~mm}$ inlet and $\varphi 1 \mathrm{~mm}$ outlet (Fig. S1†).

\section{Analysis by FESEM, FESEM-EDS, FT-IR and AFM of MPS-SH SAM-modified nanowires in a microchannel}

Top and cross-sectional images of $\mathrm{ZnO}$ nanowires and the substrate with Au-coated nanowires in the microchannel were obtained using a field emission scanning electron microscope (FESEM) (Supra40vp, Carl Zeiss AG). The diameter, length and orientation angle of each nanowire were measured from FESEM images using the image processing software ImageJ.

Elemental mappings of $\mathrm{ZnO}$ nanowire and Au-coated nanowire substrates were performed using FESEM (JSM$7610 \mathrm{~F}$, Jeol) equipped with the energy dispersive X-ray spectrometer (EDS) function. An accelerating voltage of $15 \mathrm{kV}$ was chosen to obtain the cross-sectional images. The images were $512 \times 384$ pixels, and the delay time for each pixel was $0.1 \mathrm{~ms}$. The images were integrated for 100 cycles. $\mathrm{Zn} \mathrm{K \alpha}$ (1.012 eV), O K $\alpha(0.525 \mathrm{eV})$ and $\mathrm{Au} \mathrm{M} \alpha(2.120 \mathrm{eV})$ were chosen to construct each image.

Fourier transform infrared (FT-IR) spectra of solid state MPC-SH solution containing $100 \mathrm{mM}$ MPC-SH in pure water and MPC-SH SAM-modified nanowire substrate were measured using an FT-IR spectrometer (Nicolet iS50, Thermo Fisher Scientific Inc.) equipped with a built-in diamond-attenuated total reflection (ATR) module. ATR measurements were performed for solid state MPC-SH and $100 \mathrm{mM}$ MPC-SH aqueous solutions. A deuterated triglycine sulfate (DTGS) detector was used for the ATR measurements with 500 scans and $4 \mathrm{~cm}^{-1}$ resolution. The substrate with MPC-SH SAM-modified nanowires was measured in the transmission mode using an HgCdTe (MCT) detector. The spectrum was obtained from 1000 scans and $4 \mathrm{~cm}^{-1}$ resolution. Transmission measurements were performed under an $\mathrm{N}_{2}$ atmosphere at room temperature. The substrate with Au-coated nanowires was used for background correction of MPC-SH SAM-modified nanowires. 
Atomic force microscopy (AFM) observation was performed using SPA400 and SPI3800N systems (Seiko Instruments Inc.) to confirm whether MPC-SH could be modified in a self-assembled monolayer. For this experiment, flat substrates with an Au layer before and after MPC-SH treatment were prepared. Imaging was conducted in the dynamic force mode using a silicon cantilever SI-DF20 (Seiko Instruments Inc.) with a resonance frequency of $125 \mathrm{kHz}$.

\section{CRP assay using MPS-SH SAM-modified nanowires in a} microchannel

Assay buffer for CRP capture was prepared by dissolving 1 $\mathrm{mM} \mathrm{CaCl} 2$ into 2-( $N$-morpholino)ethanesulfonic acid (MES) buffer (10 mM MES, $100 \mathrm{mM} \mathrm{NaCl}$, pH 5.5). The $2 \mu \mathrm{L}$ sample solution with $21 \mu \mathrm{g} \mathrm{mL} \mathrm{m}^{-1} \mathrm{CRP}$ (C-reactive protein from human plasma, Sigma-Aldrich Co. LLC.) diluted in the assay buffer was introduced into the microchannel with the MPCSH SAM-modified nanowires by using an aspirator (VACUSIP, Scitech Co., Ltd.). After $30 \mathrm{~min}$ incubation at room temperature, the microchannel was washed first with wash buffer (MES buffer, $1 \mathrm{mM} \mathrm{Ca}^{2+}$ and $0.5 \%$ Tween 20 ) and then assay buffer. Two $\mu \mathrm{L}$ primary antibody $\left(50 \mu \mathrm{g} \mathrm{mL} \mathrm{m}^{-1}\right.$, anti-C reactive protein antibody, ab31156, Abcam plc.) was introduced into the microchannel and after $30 \mathrm{~min}$ incubation, the microchannel was washed again with wash buffer and then assay buffer. Two $\mu \mathrm{L}$ secondary antibody with DyLight 650 fluorescence-labeling (50 $\mu \mathrm{g} \mathrm{mL}^{-1}$, goat anti-rabbit IgG Fc (DyLight® 650) preadsorbed, ab98465, Abcam plc.) was also introduced, incubated and washed following the same procedures as those used for the primary antibody. The microchannel was filled with assay buffer to observe it with a fluorescence microscope (ECLIPSE Ti, Nikon Co.). Fluorescence intensity was measured from fluorescence images obtained for the inside of the microchannel by the image processing software ImageJ.

\section{Results and discussion}

\section{MPC-SH SAM-modified nanowires in a microchannel}

MPC-SH SAM-modified nanowires in a microchannel were fabricated (Fig. S1 $\dagger$ ). For the $\mathrm{ZnO}$ nanowire fabrication in the microchannel, we sputtered the ZnO layer onto the microchannel bottom surface following microchannel fabrication. The nanowires were hydrothermally grown using several $\mathrm{ZnO}$ layers with various thicknesses, which are known to affect ZnO grain sizes, ${ }^{39}$ and they also acted as templates for the ZnO nanowire during hydrothermal synthesis. This allowed rough control of the $\mathrm{ZnO}$ nanowire length, diameter, and orientation angle (Fig. 2). A thicker ZnO layer (larger grain size) resulted in a shorter length and larger diameter of $\mathrm{ZnO}$ nanowires. To obtain the most oriented nanowire for a uniform Au-sputtered layer, we decided to use the $100 \mathrm{~nm} \mathrm{ZnO}$ seed layer in the following analyses. FESEM observation revealed that the ZnO nanowires inside the microchannel had an average length of $824 \mathrm{~nm}$ and a diameter of $61 \mathrm{~nm}$ (Fig. 2a-i).

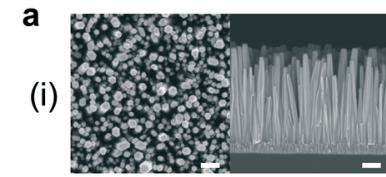

(ii)
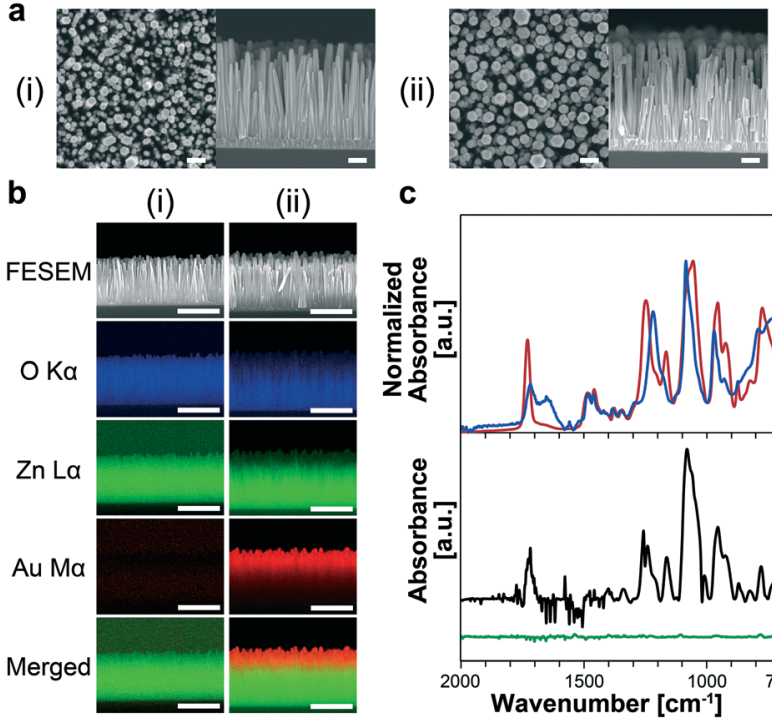

C

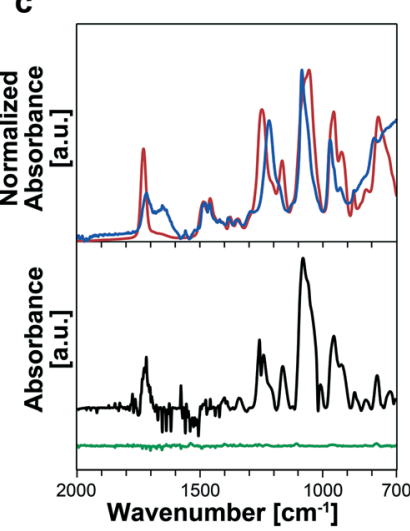

Fig. 2 (a) FESEM images of (i) $\mathrm{ZnO}$ nanowires and (ii) Au-coated $\mathrm{ZnO}$ nanowires (left images, top view; right images, cross-sectional view; scale bars, $200 \mathrm{~nm}$ ). (b) Images of elemental mapping of (i) ZnO nanowires and (ii) Au-coated $\mathrm{ZnO}$ nanowires from top to bottom: SEM, O $\mathrm{K} \alpha, \mathrm{Zn} \mathrm{L} \alpha, \mathrm{Au} M \alpha$, merged (scale bars, $1 \mu \mathrm{m}$ ). The merged images were obtained from $\mathrm{Zn} L \alpha$ (green) and $\mathrm{Au} M \alpha$ (red) images. (c) FT-IR spectra of MPC-SH solid powder (red) and MPC-SH (100 mM) in pure water (blue), MPC-SH SAM-modified nanowires (black) and MPC-SH-treated $\mathrm{ZnO}$ nanowires without Au layer (green). Both MPC-SH samples were measured by ATR, and MPC-SH SAM modified nanowires and $\mathrm{ZnO}$ nanowires were measured in the transmission mode.

After $\mathrm{Au}$ was coated onto $\mathrm{ZnO}$ nanowires (Fig. 2a-ii), elemental mappings revealed that around one third of the nanowires had an Au layer from the nanowire tips (Fig. 2b). The other two-thirds of the nanowires from the nanowire bases might have clusters of $\mathrm{Au}$ atoms, rather than a layer, and a few clusters were found at the base of the nanowires. Also, the nanowire tip diameters increased in response to increased sputtering time $(0,15,30$, and $60 \mathrm{~s})$ (Fig. S3†). These results were interpreted from sputtering behavior. ${ }^{40}$

MPC-SH SAM-modified nanowires were obtained by treating Au-coated $\mathrm{ZnO}$ nanowires with MPC-SH. The modified nanowires showed several peaks in their FT-IR spectrum (Fig. 2c). These peaks corresponded to those for MPC-SH solid powders and MPC-SH (100 mM) in pure water. Therefore, we confirmed that the Au-coated nanowires were modified by the MPC-SH layers. Some specific peaks were measured at 1716, 1255, 1077 and $953 \mathrm{~cm}^{-1}$, indicating $\mathrm{C}=\mathrm{O}$, $\mathrm{P}=\mathrm{O}$, and two $\mathrm{P}-\mathrm{O}-\mathrm{C}$ stretching modes, respectively. The $\mathrm{C}=\mathrm{O}$ stretching mode was assigned to the ester group, and the $\mathrm{P}=\mathrm{O}$ and $\mathrm{P}-\mathrm{O}-\mathrm{C}$ stretching modes originated from the phosphate ester functional group. On the other hand, $\mathrm{ZnO}$ nanowires treated with MPC-SH had no distinct peaks from MPC-SH in the FT-IR spectrum due to lack of Au layers (Fig. 2c). Therefore, we concluded that MPC-SH was selectively attached via Au-S linkage on the Au-coated nanowires.

The AFM measurement of flat substrates with an Au layer before and after MPC-SH treatment revealed that the Au layer 
had many grains due to sputtering. In addition, the sizes of the $\mathrm{Au}$ grains of $\mathrm{Au} / \mathrm{ZnO} / \mathrm{Si}$ substrates before and after MPCSH modification were almost identical (Fig. S4 $\dagger$ ), suggesting that MPC-SH modification resulted in a molecularly thin layer. Additionally, the root mean square (RMS) values of the substrates before and after modification were 1.35 and 1.24 $\mathrm{nm}$, respectively. We understand that the minor change in RMS values before and after modification is derived from the few occurrences of MPC-SH aggregations on the Au surface. These results support the successful formation of MPC-SH $\mathrm{SAM}$ on the Au-coated nanowires.

\section{CRP assay using MPC-SH SAM-modified nanowires in a microchannel}

The MPC-SH SAM-modified nanowires enabled selective capture of CRP in the presence of calcium ions. We introduced CRP and calcium ions, followed by labelling with primary antibodies and secondary antibodies with fluorescence dyes

a

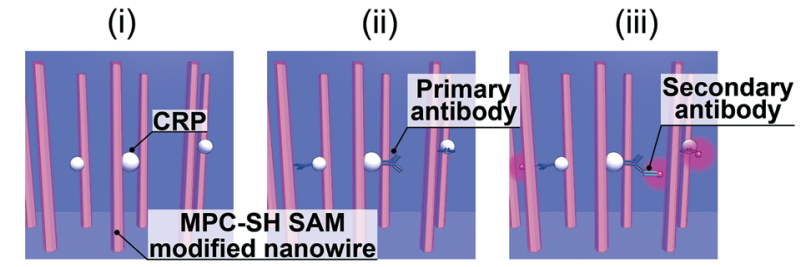

b (i)

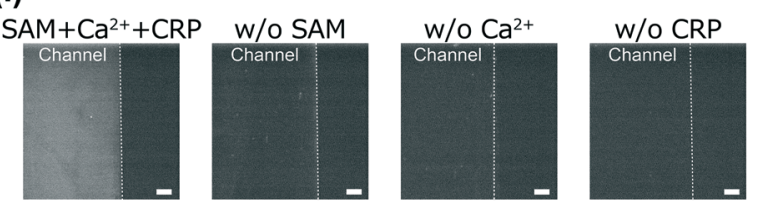

(ii)

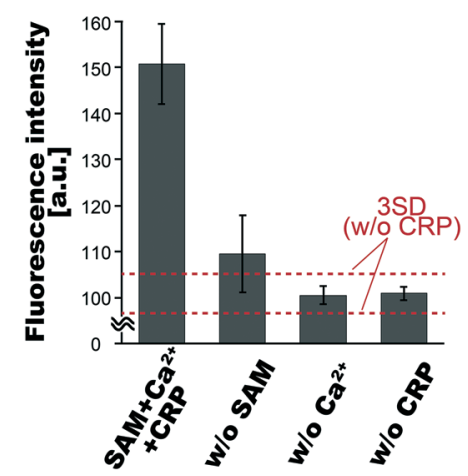

Fig. 3 (a) Schematic illustration of CRP assay using MPC-SH SAMmodified nanowires in a microchannel: (i) CRP introduction and capture onto the nanowires in the presence of calcium ions; (ii) CRP tagged by primary antibody; and (iii) fluorescent labelling by secondary antibody. (b) (i) Fluorescence images (scale bars, $200 \mu \mathrm{m}$ ) and (ii) fluorescence-based evaluation of CRP capture by the SAM-modified nanowires $\left(S A M+\mathrm{Ca}^{2+}+\mathrm{CRP}\right)$, the $\mathrm{Au}$-coated nanowires (w/o SAM), the SAM-modified nanowires without calcium ions $\left(\mathrm{w} / \mathrm{o} \mathrm{Ca}^{2+}\right)$, and the SAM-modified nanowires without CRP (w/o CRP). In the fluorescencebased evaluation, the background was defined as w/o CRP. The horizontal red dotted lines show the signal level at 3 standard deviations above the background (3SD). Error bars show the standard deviation for a series of measurements $(N=3)$. for the CRP assay (Fig. 3a). The CRP assay was performed under four sets of conditions: (I) with SAM, $\mathrm{Ca}^{2+}$ and CRP; (II) without SAM; (III) without $\mathrm{Ca}^{2+}$; and (IV) without CRP (Fig. 3b). We confirmed the increase in fluorescence intensity for (I) and (II). However, (II) represents the original Au-coated nanowires, which should inevitably cause nonspecific adsorption of CRP on their surfaces due to lack of antifouling ability. From the low fluorescence intensities at (III) and (IV), we inferred that the MPC-SH SAM-modified nanowires did not exhibit CPR recognition ability in the absence of calcium ions, and nonspecific adsorption of CRP was also suppressed. ${ }^{23,25,28}$ Therefore, only condition (I) (with SAM, $\mathrm{Ca}^{2+}$ and CRP) exhibited successful CRP assay.

The suppression of nonspecific adsorption by the MPC-SH SAM-modified nanowires is due to the PC groups. The zwitterion in the PC group forms a strong hydration layer via electrostatic interactions, and this hydration layer enables the repulsion of proteins at the SAM/solution interface. ${ }^{41,42}$ Furthermore, the PC group provides recognition of CRP with the aid of calcium ions. The PC-binding site in CRP is coordinated and activated by two calcium ions. ${ }^{27}$ This leads CRP to be recognized by the PC groups in MPC-SH. ${ }^{28}$ Due to these reasons, MPC-SH SAM enables the suppression of nonspecific absorption and promotion of CRP recognition in the presence of calcium ions.

\section{Conclusions}

Herein, we demonstrated biomolecular recognition of a target protein on nanowire surfaces modified by MPC-SH SAM instead of primary antibodies. MPC-SH SAM was chemically attached to the Au-coated nanowire surfaces by a single process. The surface modification enabled the recognition of CRP with calcium ions and reduced nonspecific adsorption. We believe that the presented methodology will provide a new strategy in the development of nanostructure-embedded biosensors without the use of antibodies. To make full use of the potential of SAM modification proposed in this paper, complete antibody-free detection is very important because antibodies for fluorescence-based labelling were used for detection in this paper. Recently, nanowire-embedded biosensors have been developed for label-free detection, ${ }^{8,9,15,20}$ and the SAM-modified nanowires proposed in this paper have the potential to be utilized in the biosensors for biomolecular recognition on the nanowire surfaces, which enables completely label-free detection without the use of antibodies. This paper demonstrates the application potential for labelfree detection via a nanowire-embedded biosensor, and the key achievement is biomolecular recognition on the nanowire surface modified by MPC-SH SAM instead of primary antibodies.

\section{Author contributions}

T. S., T. Yasui, A. Y., T. G., M. H., T. Yanagida, N. K., M. K., K. N., Y. M., T. K., and Y. B. planned and designed 
experiments. T. S., A. Y., T. G. and M. H. performed experiments. T. S., T. Yasui, A. Y., T. G., M. H., N. K. analyzed data. T. S., T. Yasui, T. G., T. Yanagida, M. K., K. N. wrote the paper.

\section{Conflicts of interest}

There are no conflicts of interest to declare.

\section{Acknowledgements}

This research was supported by the Grant-in-Aid for JSPS Research Fellow (17J05751), PREST (JPMJPR151B, JPMJPR16F4), JST, the JSPS Grant-in-Aid for Young Scientists (A) 17H04803, the ImPACT Program of the Council for Science, Technology and Innovation (Cabinet Office, Government of Japan), and the JSPS Grant-in-Aid for Scientific Research (A) 16H02091.

\section{Notes and references}

1 S. H. Gellman, Chem. Rev., 1997, 97, 1231.

2 P. Chen, N. T. Huang, M. T. Chung, T. T. Cornell and K. Kurabayashi, Adv. Drug Delivery Rev., 2015, 95, 90.

3 Y. Song, P. Chen, M. T. Chung, R. Nidetz, Y. Park, Z. Liu, W. McHugh, T. T. Cornell, J. Fu and K. Kurabayashi, Nano Lett., 2017, 17, 2374.

4 P. Yánez-Sedeño, S. Campuzano and J. M. Pingarrón, Appl Mater Today, 2017, 9, 276.

5 A. Zamora-Gálvez, E. Morales-Narváez, C. C. MayorgaMartinez and A. Merkoçi, Appl Mater Today, 2017, 9, 387.

6 M. Pumera, TrAC, Trends Anal. Chem., 2017, 93, 1.

7 Y. Xing, A. Wyss, N. Esser and P. S. Dittrich, Analyst, 2015, 140, 7896.

8 J.-i. Hahm and C. M. Lieber, Nano Lett., 2004, 4, 51.

9 N. Gao, T. Gao, X. Yang, X. Dai, W. Zhou, A. Zhang and C. M. Lieber, Proc. Natl. Acad. Sci. U. S. A., 2016, 113, 14633.

10 J. Li, G. He, H. Ueno, C. Jia, H. Noji, C. Qi and X. Guo, Nanoscale, 2016, 8, 16172.

11 G. Pampalakis and S. O. Kelley, Analyst, 2009, 134, 447.

12 Y.-Q. Li, B. Zhu, Y. Li, W. R. Leow, R. Goh, B. Ma, E. Fong, M. Tang and X. Chen, Angew. Chem., Int. Ed., 2014, 53, 5837.

13 K. Zhu, Y. Zhang, Z. Li, F. Zhou, K. Feng, H. Dou and T. Wang, Sensors, 2015, 15, 19225.

14 C. Seokheun and C. Junseok, J. Micromech. Microeng., 2010, 20, 075015.

15 C. Y. Hsiao, C. H. Lin, C. H. Hung, C. J. Su, Y. R. Lo, C. C. Lee, H. C. Lin, F. H. Ko, T. Y. Huang and Y. S. Yang, Biosens. Bioelectron., 2009, 24, 1223.

16 R. Ahmad, N. Tripathy, J. H. Park and Y. B. Hahn, Chem. Commun., 2015, 51, 11968.

17 S. Rahong, T. Yasui, N. Kaji and Y. Baba, Lab Chip, 2016, 16, 1126.
18 X. Dai, W. Zhou, T. Gao, J. Liu and C. M. Lieber, Nat. Nanotechnol., 2016, 11, 776.

19 J. Liu, T. M. Fu, Z. Cheng, G. Hong, T. Zhou, L. Jin, M. Duvvuri, Z. Jiang, P. Kruskal, C. Xie, Z. Suo, Y. Fang and C. M. Lieber, Nat. Nanotechnol., 2015, 10, 629.

20 Y. Cui, Q. Wei, H. Park and C. M. Lieber, Science, 2001, 293, 1289.

21 T. Yasui, T. Yanagida, S. Ito, Y. Konakade, D. Takeshita, T. Naganawa, K. Nagashima, T. Shimada, N. Kaji, Y. Nakamura, I. A. Thiodorus, Y. He, S. Rahong, M. Kanai, H. Yukawa, T. Ochiya, T. Kawai and Y. Baba, Sci. Adv., 2017, 3, e1701133.

22 A. Choi, K. Kim, H.-I. Jung and S. Y. Lee, Sens. Actuators, B, 2010, 148, 577.

23 T. Goda, M. Tabata, M. Sanjoh, M. Uchimura, Y. Iwasaki and Y. Miyahara, Chem. Commun., 2013, 49, 8683.

24 J. E. Volanakis and K. W. A. Wirtz, Nature, 1979, 281, 155.

25 Y. Iwasaki, T. Kimura, M. Orisaka, H. Kawasaki, T. Goda and S.-i. Yusa, Chem. Commun., 2014, 50, 5656.

26 T. Goda, M. Toya, A. Matsumoto and Y. Miyahara, ACS Appl. Mater. Interfaces, 2015, 7, 27440.

27 A. Agrawal, S. Lee, M. Carson, S. V. Narayana, T. J. Greenhough and J. E. Volanakis, J. Immunol., 1997, 158, 345.

28 T. Goda, P. Kjall, K. Ishihara, A. Richter-Dahlfors and Y. Miyahara, Adv. Healthcare Mater., 2014, 3, 1733.

29 P. Buchegger and C. Preininger, Anal. Chem., 2014, 86, 3174.

30 C. Gabay and I. Kushner, N. Engl. J. Med., 1999, 340, 448.

31 N. Rifai, R. P. Tracy and P. M. Ridker, Clin. Chem., 1999, 45, 2136.

32 P. M. Ridker, C. H. Hennekens, J. E. Buring and N. Rifai, $N$. Engl. J. Med., 2000, 342, 836.

33 I. S. Ockene, C. E. Matthews, N. Rifai, P. M. Ridker, G. Reed and E. Stanek, Clin. Chem., 2001, 47, 444.

34 P. M. Ridker, Circulation, 2001, 103, 1813.

35 M. B. Pepys and G. M. Hirschfield, J. Clin. Invest., 2003, 111, 1805.

36 P. Libby and P. M. Ridker, Am. J. Med., 2004, 116, 9.

37 J. Joo, B. Y. Chow, M. Prakash, E. S. Boyden and J. M. Jacobson, Nat. Mater., 2011, 10, 596.

38 Y. He, T. Yanagida, K. Nagashima, F. Zhuge, G. Meng, B. Xu, A. Klamchuen, S. Rahong, M. Kanai, X. Li, M. Suzuki, S. Kai and T. Kawai, J. Phys. Chem. C, 2013, 117, 1197.

39 W. Y. Wu, C. C. Yeh and J. M. Ting, J. Am. Ceram. Soc., 2009, 92, 2718.

40 Y. Liu, W. Chen, S. Wei and W. Gao, RSC Adv., 2014, 4, 30658.

41 S. Chen, J. Zheng, L. Li and S. Jiang, J. Am. Chem. Soc., 2005, 127, 14473.

42 Y. He, J. Hower, S. Chen, M. T. Bernards, Y. Chang and S. Jiang, Langmuir, 2008, 24, 10358. 